\title{
Inner Core Anisotropic Structure Using Global Coda-correlation Wavefield
}

\author{
Thuany Costa de Lima ${ }^{1}$, Hrvoje Tkalčić ${ }^{1}$, and Lauren Waszek ${ }^{2,3}$ \\ ${ }^{1}$ Research School of Earth Sciences, The Australian National University, 142 Mills Road, Canberra, ACT, 2601, \\ Australia \\ ${ }^{2}$ College of Science and Engineering, James Cook University, Douglas, QLD, 4811, Australia \\ ${ }^{3}$ Department of Physics, New Mexico State University, Las Cruces, NM, 88003, United States of America \\ Copyright 2021, SBGf - Sociedade Brasileira de Geofísica. \\ This paper was prepared for presentation during the $17^{\text {th }}$ International Congress of the Brazilian Geophysical Society held in Rio de Janeiro, Brazil, $8-11$ November 2021 (Online Event). \\ Contents of this paper were reviewed by the Technical Committee of the $17^{\text {th }}$ International Congress of the Brazilian Geophysical Society and do not necessarily represent any position of the \\ SBGf, its officers or members. Electronic reproduction or storage of any part of this paper for commercial purposes without the written consent of the Brazilian Geophysical Society is
} prohibited.

A major challenge in exploring the anisotropic structure of the Earth's inner core (IC) is the limitation of its volumetric sampling, restricted by the uneven distribution of earthquakes and receivers. The poor coverage of ray paths sensitive to the deep IC, especially in the polar direction, leads to uncertainties in the strength and orientation of its anisotropy. Yet, improved constraints on the anisotropy are required to understand the crystallographic structure of iron in the IC, and the evolution of its solidification and deformation processes. In this study, we investigate the IC anisotropy properties using the recently-developed technique based on Earth's coda-correlation wavefield constructed from the late coda of large earthquakes. We perform travel-time analysis of $\mathrm{I}^{*}$, a correlation feature sensitive to the Earth's IC that resembles PKIKPPKIKP compressional waves in the direct seismic wavefield. $12^{*}$ is a mathematical manifestation of cross-terms of multiple seismic phases with similarities in slowness, and therefore, substantially different from PKIKPPKIKP. Here, we show that the travel time of these core-correlated phases is highly dependent on the location of the earthquakes and receiver pairs, and this guides our selection of data. We then present a travel-time analysis of correlation stacks obtained from podal-antipodal source-station geometry. The travel time variability of $12^{*}$ with the direction within the IC supports a model of cylindrical anisotropy with a slow axis at $\xi=55^{\circ}$, with azimuthal variations, where $\xi$ is the angle between the ray path in the IC and the Earth's rotation axis. This observation indicates a distinct anisotropic pattern in the deepest portion of the IC and thus confirms the existence of the innermost inner core, proposed almost 20 years ago. 